\title{
Crystallographic insight into enhanced catalytic activity of carbonic anhydrase II using "activating" ligands
}

\author{
Avni Bhatt $^{\mathrm{a}}$, Marc A. Ilies ${ }^{\mathrm{b}}$, Robert McKenna $^{\mathrm{a}}$ \\ a Department of Biochemistry and Molecular Biology, College of Medicine, \\ University of Florida, Gainesville FL, USA \\ ${ }^{b}$ Department of Pharmaceutical Sciences, School of Pharmacy, Temple \\ University, Philadelphia PA, USA
}

Carbonic anhydrase is a zinc metalloenzyme whose structure and function plays an important role in therapeutic design. In particular, carbonic anhydrase II (CA II) is the fastest isoform of the enzyme, rapidly catalyzing the interconversion of carbon dioxide to bicarbonate and a proton. This reaction is essential for $\mathrm{pH}$ regulation and is instrumental in the function of various neurotransmitters, such as glutamate, GABA and dopamine. In the hydration direction, the enzyme functions by utilizing a catalytic zinc-bound hydroxide for the formation of bicarbonate, leaving a zinc-bound water. To convert the enzyme back to its active form, a proton bound to the zinc must be released via a proton wire and expelled from the active site. A class of synthesized small-molecule ligands has shown to significantly increase CA Il's catalytic conversion rate, and have previously demonstrated a strong nootropic effect in rat models, improving memory, learning, and cognition. Since this is the rate-limiting step of the reaction, it is believed that these activating ligands function by assisting in the proton-transfer step, serving as a secondary site for proton release. However, the precise mechanism of this activation was previously unknown. We present the crystal structure of CA II in complex with one such activating ligand, depicting the precise binding motif in the active site to a resolution of $1.6 \mathrm{~A}$. This structure validates the postulated method of activation through an enhanced proton shuttling mechanism and provides insight into the endogenous catalytic function of the enzyme. Furthermore, the network of active site water molecules can be closely studied using neutron diffraction to understand the differences in solvent organization with the addition of the activating ligand. This work will vastly impact the design of CA activating ligands for future therapeutic applications. 